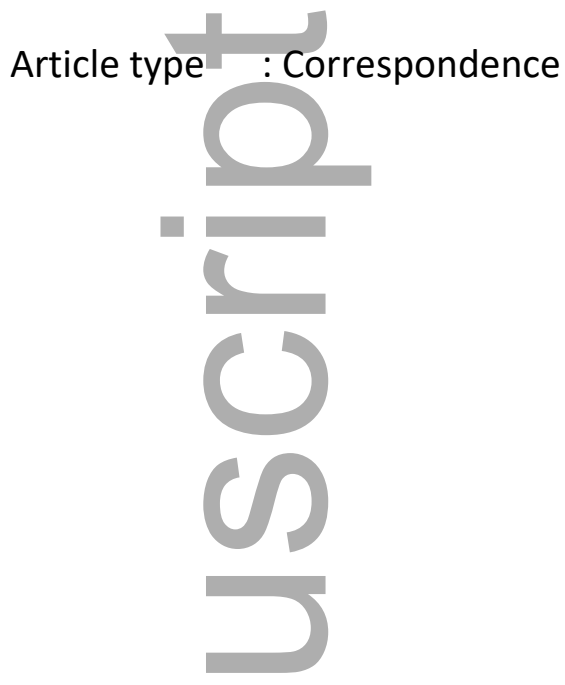

TITLE: Robotic abdominoperineal resection, posterior vaginectomy and sacrectomy - A video vignette

\title{
AUTHORS and their role:
}

Dr Jessica Rahme - Editing of video and audio

Dr Amrish Rajkomar - Surgical assistant

Dr Jose Tomas Larach - Editing of video and transcript

Mr Ramin Shayan - Plastic surgeon performing IGAM flap

Mr Joseph Kong - Surgical assistant

ProfAlexander Heriot - Editing of video and transcript

Mr Satish Warrier - Primary surgeon

VIDEO DURATION - 10 minutes

This is the author manuscript accepted for publication and has undergone full peer review but has not been through the copyediting, typesetting, pagination and proofreading process, which may lead to differences between this version and the Version of Record. Please cite this article as doi: $10.1111 /$ CODI.15427

This article is protected by copyright. All rights reserved 


\section{CONFLICTS OF INTEREST -None Declared}

\section{Transcript}

In this video we describe our method for performing a robotic abdominoperineal resection, posterior vaginectomy and distal sacrectomy.

We present the case of a $75 y$ female with a recurrence of her rectal cancer on a background of extensive abdominal operations. She was initially diagnosed in 2014 at which time she underwent chemoradiotherapy. This was followed by two liver resections and a Hartmann's procedure. She received radiotherapy in 2018 for a metastatic right upper lobe lung lesion. She has also had a hysterectomy and cholecystectomy and has hypothyroidism and hypertension.

In March 2020, surveillance revealed elevated CEA levels and an FDG PET avid lesion at the apex of her rectal stump. Flexible sigmoidoscopy confirmed this lesion. MRI showed a $1.4 \times 1.4 \times 0.7 \mathrm{~cm}$ soft tissue thickening at the apex of the rectal stump extending into the posterior vaginal wall and presacral fascia.

After MDM (multidisciplinary meeting) discussion, it was decided that the patient would undergo robotic abdominoperineal resection with en bloc posterior vaginectomy and distal sacrectomy. She would be for consideration of right upper lobe lobectomy after recovery from this procedure. Our method is as follows:

Port configuration.

There are four $8 \mathrm{~mm}$ ports, a gel-point mini and an assistant port.

Step 1 is adhesiolysis for port placement.

The extensive adhesions reduce the working space considerably. As such, only two instruments are used initially. We go underneath the omentum and dissect small bowel off the anterior abdominal wall.

Step 2 is adhesiolysis to isolate the stoma.

Four ports are used and progress is made with ongoing small bowel adhesiolysis. Despite the difficult adhesiolysis, progress is made safely. Therefore, we did not to convert to open surgery. Additionally, adhesiolysis would likely be equally difficult post conversion. 
Step 3 is adhesiolysis of small bowel from the pelvis.

Step 4 is ureterolysis.

Our preferred method is ureteric stent placements with ICG. However in this case the stents were displaced pre-operatively. After completion of ureterolysis the lateral pelvic adhesions are largely cleared. At this point, the anterior pelvic adhesions are approached. The small bowelcan now be removed from the pelvis.

Step 5 is demarcation of resection margins.

First the poster margin on the sacrum is marked. The anterior margin on the posterior vaginal wall at the level of the cervix is then marked, guided by a vaginal probe. Once we are satisfied that these levels are safe the sacrum is divided below the s4 foramina.

Step 6 is to complete the dissection circumferentially, including down to the bone posteriorly.

The posterior wall of the vagina is dissected en-bloc with the rectum. A McCartney tube is used to maintain pneumoperitoneum. The lateral resection margins are then demarcated. At this level there is very low risk of injury to the ureter. The anterior portion of the dissection is then complete.

Step 7 is cutting of sacrum.

We have chosen to use the Misonix bone scalpel. It uses advanced ultrasonic technology to divide bone. The blade is introduced through the gel-point mini. It is controlled by a foot pedal at the robot console. Downward pressure is applied by the assistant. A see-saw pattern is used to divide bone and it simultaneously seal the vessels.

Step 8 is division of soft tissue posterior to the sacrum.

We use scissors to separate the soft tissue beyond the sacral bone into the ischioanal space. The post sacral fat becomes visible and is continuous with the ischioanal fat. The bone can be seen to be clearly separated.

The abdominal robotic resection component of the operation is now complete. A size 15 Blakes drain is placed in the pelvis. Perineal resection is subsequently completed and is directed posterior to the sacrum so that it is removed en bloc with the rectum and posterior vaginal wall. The pelvic cavity post resection can then be seen.

Our plastic surgeon colleagues then did an Inferior Gluteal Artery Myocutaneous Island Transposition Flap Reconstruction. 
The total operating time was 7 hours with $100 \mathrm{mls}$ of intra-op blood loss. There were no intra or post operative complications. Colostomy function resumed on day 3 post operatively.

Flap care included 2 weeks of side-to-side and lying prone, with graduated supine lying and frequent review. The LOS was 20 days at which point she was discharged to rehabilitation with IDC in-situ due to low patient confidence.

There was follow up by the plastic surgeons with Cystoscope $r / v$ vaginal reconstruction at 8 weeks. Colorectal follow up was a clinic appointment at 4 weeks, CT abdo/pelvis at 6 months and colonoscopy at 12 months.

Pathology revealed a T3 moderately differentiated adenocarcinoma involving full thickness of the rectum and into the adventitia. There was no involvement of the vaginal wall. The specimen was $44 \mathrm{~mm}$ in maximum dimension and there were no lymph nodes.

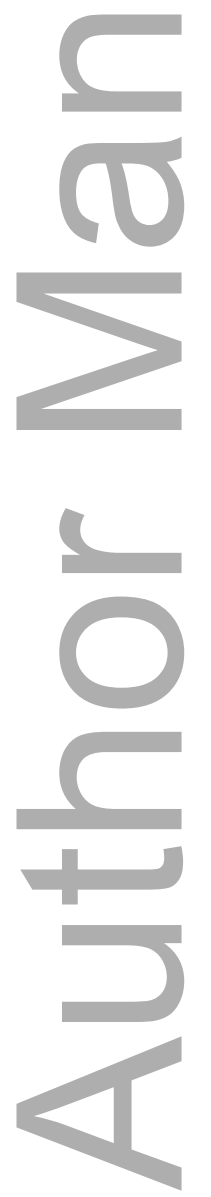




\section{VIDEO VIGNETTE}

In this video, we describe our method for performing a robotic abdominoperineal resection, posterior vaginectomy and sacrectomy. We present the case of a 75 year old female with a recurrence of her rectal cancer on a background of extensive abdominal operations. She was initially diagnosed six years ago and has undergone two liver resections and a Hartmann's procedure. Surveillance six years post diagnosis revealed a new lesion at the apex of her rectal stump and MRI suggested extension into the posterior vaginal wall and presacral fascia. After multidisciplinary meeting discussion, it was decided that the patient would undergo robotic abdominoperineal resection with en bloc posterior vaginectomy and distal sacrectomy followed by an inferior gluteal artery myocutaneous island transposition flap reconstruction. The procedure included extensive adhesiolysis. A Misonix bone scalpel was used to divide the sacrum which has not previously been described in robotic surgery. This device uses advanced ultrasonic technology to divide the bone. Pathology revealed a T3 moderately differentiated adenocarcinoma involving full thickness of the rectum and no involvement of the vaginal wall. There were no short or longterm complications.

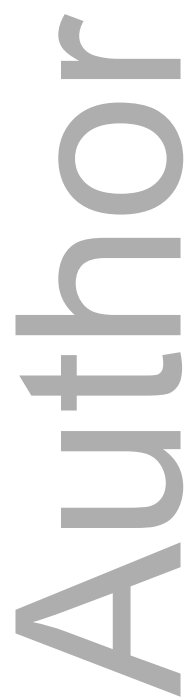




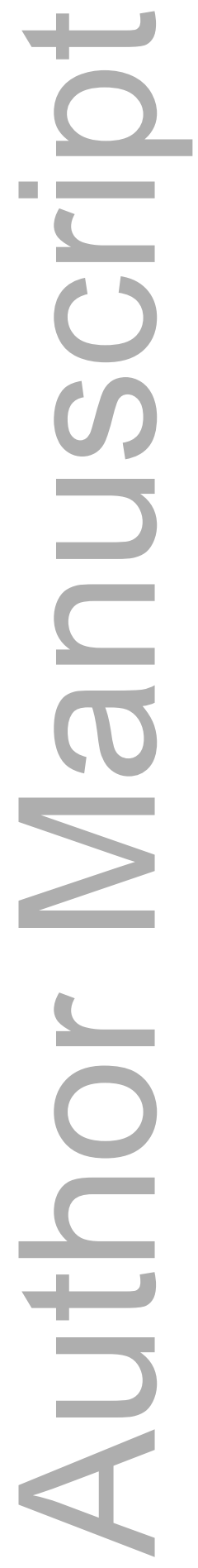

This article is protected by copyright. All rights reserved 


\section{University Library}

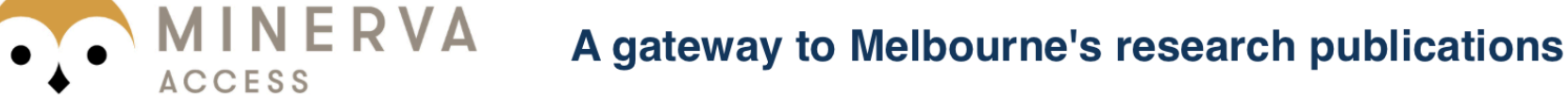

Minerva Access is the Institutional Repository of The University of Melbourne

\section{Author/s:}

Rahme, J;Rajkomar, A;Larach, JT;Shayan, R;Kong, JCH;Heriot, A;Warrier, S

Title:

Robotic abdominoperineal resection, posterior vaginectomy and sacrectomy - a video vignette

Date:

2020-11-18

\section{Citation:}

Rahme, J., Rajkomar, A., Larach, J. T., Shayan, R., Kong, J. C. H., Heriot, A. \& Warrier, S. (2020). Robotic abdominoperineal resection, posterior vaginectomy and sacrectomy - a video vignette. COLORECTAL DISEASE, 23 (2), pp.561-562. https://doi.org/10.1111/ codi. 15427.

Persistent Link:

http://hdl.handle.net/11343/276627 